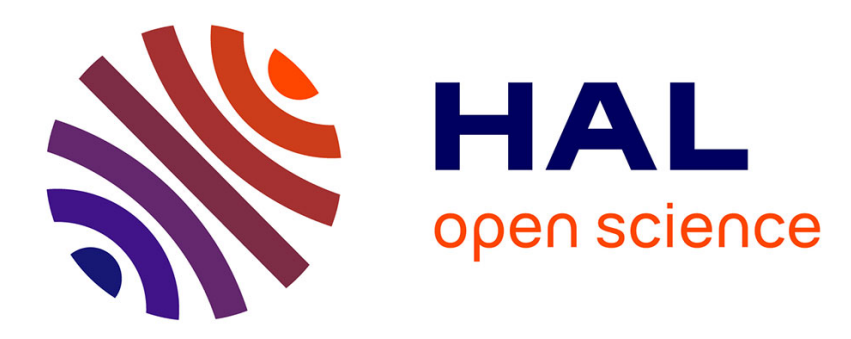

\title{
Fine-Structure Constant from Golden Ratio Geometry
}

Michael Sherbon

\section{To cite this version:}

Michael Sherbon. Fine-Structure Constant from Golden Ratio Geometry. International Journal of Mathematics and Physical Sciences Research, 2018. hal-01756341

\section{HAL Id: hal-01756341 https://hal.science/hal-01756341}

Submitted on 1 Apr 2018

HAL is a multi-disciplinary open access archive for the deposit and dissemination of scientific research documents, whether they are published or not. The documents may come from teaching and research institutions in France or abroad, or from public or private research centers.
L'archive ouverte pluridisciplinaire HAL, est destinée au dépôt et à la diffusion de documents scientifiques de niveau recherche, publiés ou non, émanant des établissements d'enseignement et de recherche français ou étrangers, des laboratoires publics ou privés. 


\title{
Fine-Structure Constant from Golden Ratio Geometry
}

\author{
Michael A. Sherbon \\ Case Western Reserve University Alumnus \\ E-mail:michael.sherbon@case.edu
}

January 24, 2018

\begin{abstract}
After a brief review of the golden ratio in history and our previous exposition of the finestructure constant and equations with the exponential function, the fine-structure constant is studied in the context of other research calculating the fine-structure constant from the golden ratio geometry of the hydrogen atom. This research is extended and the fine-structure constant is then calculated in powers of the golden ratio to an accuracy consistent with the most recent publications. The mathematical constants associated with the golden ratio are also involved in both the calculation of the fine-structure constant and the proton-electron mass ratio. These constants are included in symbolic geometry of historical relevance in the science of the ancients.
\end{abstract}

Keywords fine-structure constant, dimensionless physical constants, history of mathematics, golden ratio, sacred geometry, Fibonacci sequence, mathematical constants, fundamental physics.

\section{Introduction}

As Willem Witteveen states in his book The Great Pyramid of Giza, "Every expression of the golden mean, which includes: number, rectangle, triangle, spiral and frequency, is encoded in the design of the Great Pyramid and illustrates the importance of the ratio in the universe in which we belong." [1]. He also states, "The golden ratio, as well as the Great Pyramid as an expression of it, is an important key to our universe containing the Earth and the Moon" [1] and that "the ratio between the Earth and the Moon is in fact the basis for the mathematical concept of 'squaring the circle' ..." [1]. Marja de Vries states, "The Golden Ratio defines the squaring of a circle .... According to some, in ancient Egypt, this mathematical mystery was encoded in the measurements of the Great Pyramid of Giza." [2]. Continuing with her general theme of 
universal laws and wholeness, de Vries says, "In short, the idea dawns that the one universal principle ... embodiment of the Principle of Least Action ... indeed seems to be the Golden Ratio Spiral.” [2]. Richard Heath has another description, “The Golden Mean was considered a fundamental constant by the Egyptians and the fundamental division of the whole into two parts." [3]. Mario Livio says, "In fact, it is probably fair to say that the Golden Ratio has inspired thinkers of all disciplines like no other number in the history of mathematics." [4].

Alexey Stakhov explains that "one of the most important trends in the development of modern science ... is very simple: a return to the 'harmonic ideas' of Pythagoras and Plato (the 'golden ratio' and Platonic solids), embodied in Euclid's 'Elements"' [5], also see [6]. As stated previously, "The golden ratio is an approximate harmonic of the Planck length in meters and harmonics of fundamental units have a geometric basis in ancient metrology." [7, 8]. Further consideration on the nature of the golden section is given by Scott Olsen [9]. Fundamental modern applications are suggested by David Haight, "There is considerable evidence that the golden proportion is the foundation for the unification of mathematics and physics." [10], also see Gazalé [11]. In the description of Eckhart Schmitz, "Mathematics is a universal language and it would be fitting to examine the Great Pyramid in this language to derive its meaning. It has been suggested that the Great Pyramid is a repository of ancient knowledge." [12].

Thousands of years ago the ancients had an advanced mathematical understanding of universe that is revealed in many sources. There is a consistent link to knowledge of the golden mean, but the way in which the ancients were able to formulate and use this information speaks of a technical grasp of the subject that exceeds what we know about it in the present day. -Alison Primrose [13].

David Haight says, "The golden proportion is the only one in which its (legato) addition and (staccato) multiplication of itself are equivalent. It is both an arithmetic and a geometrical progression, two sides of the same coin (another "two that are one"), and is the basis of logarithms and exponentials (logarithms transform multiplication into addition, and exponentials transform addition into multiplication)." [10].

In The Essence of the Cabalah, William Eisen describes the fundamental geometry of what he described as the "Golden Apex of the Great Pyramid" [14]. Eisen's description and interpretation of Euler's identity [15]-[17], $\exp (i \pi)+1=0$ ("this most compact and mysterious formula" that "Richard Feynman referred to as "the most remarkable formula in mathematics," [18]) in relation to the Great Pyramid shows a drawing of four curves of $e^{x}$ from $x=0$ to $x=\pi$, one curve on each side and labelled the "Graphical Representation of the Exponential Function to the Base e."

$$
e^{x}=\exp (x)=\sum_{n=0}^{\infty} \frac{x^{n}}{x !}=\lim _{n \rightarrow \infty}\left(1+\frac{x}{n}\right)^{n} .
$$

In addition to the exponential function [19, 20], Euler's formula is $\exp (i x)=\cos x+i \sin x$ and in hyperbolic terms $\exp (x)=\cosh x+\sinh x$. Eisen was asking himself how the ancient Egyptians could know so much about this and how it supports his effort to understand the role of imaginary and complex numbers in the geometry of the Great Pyramid [14, 15]. The measures he found in this model with exponential curves align with other traditional measures found in the Great Pyramid design, including the golden ratio, also given as $\phi=\exp (i \pi / 5)+\exp (-i \pi / 5)$. 
These exponential curves could be considered as having the esoteric properties of an alchemical vessel as Steven Rosen explains that they "possess the feature of curving back into themselves." [21]. Wolfgang Pauli's $i$ ring, part of his World Clock vision [22], was "used in his description of microphysical spin" [21]. Rosen quotes Pauli, "The ring with the $i$ is the unity beyond particle and wave, and at the same time the operation that generates either of these." [21]. Also see the following references for Pauli and the spin concept development [23]-[29].

The exponential growth function is the simplest possible differential equation, the unique solution to $d y / d x=y ; y(0) 1$ and the most primitive (prime) derivative in which state and rate, form and function, symmetry and dynamism, being and becoming, structure and process, the integral and the derivative, the evolute and the involute, the 'splice' and the 'slice' and the squaring of a root and the extraction of a root are the same. -David Haight [30].

Dividing the sides of his mathematical model for the Great Pyramid by $\pi$ lengths results in a small square in the center called the Golden Apex, the geometry and symmetry thought to be associated with the generation of the four fundamental forces of nature [14, 15]. Eisen provides a description of the dimensions formed by the exponential function and the Golden Apex square,

$$
A=e^{\pi}-7 \pi-1 \simeq \sqrt{2} / 3 \pi \simeq 0.1495 .
$$

$A$ is the side length of the Golden Apex square. $\sqrt{A} \simeq e / 7$ and $A+1=e^{\pi}-7 \pi \simeq R \simeq 1.152$, radius of the regular heptagon with side one. The heptagon is traditionally associated with the geometry of 'squaring the circle'. The $\sin (2 \pi A) \simeq \phi / 2$, where $\phi$ is the golden ratio [31]. The $\tan (2 \pi A) \simeq 1+\sqrt{A} \simeq K / 2 \pi$, see Eq. (7) [32]. The polygon circumscribing constant is $K \simeq 2 \tan (3 \pi / 7) \simeq \phi^{2} / 2 A$, see Eq. (7) discussion and $2 A \simeq \cos \sqrt{\phi} \simeq \sqrt{2 / 7 \pi}$ [7, 32, 33]. $A$ is also the reciprocal harmonic of Newton's gravitational constant. Also, $A \simeq \ln (\sqrt{\phi}) / \phi \simeq$ $3 / e^{3} \simeq \tan ^{2}\left(e^{-1}\right)$ and $\ln \left(A^{-1}\right) \simeq \pi / \sqrt{e} \simeq \sqrt{1+\sqrt{7}} \simeq 6 / \pi \simeq \sqrt{1+\phi^{2}}$. The regular heptagon radius, $R=\csc (\pi / 7) / 2 \simeq \phi / \sqrt{2} \simeq \cot ^{2} \alpha^{-1}$ and $2 \pi R \simeq 1 / \phi \sqrt{\alpha} . R A \simeq 2 \sqrt{\alpha}$, where $\alpha$ is the fine-structure constant, see the Eq. (7) discussion below [7, 32, 33].

$$
R A \simeq \sqrt{\phi} / e^{2} \simeq \ln (\pi / \sqrt{7}) \simeq \sqrt{7 / \pi} / K .
$$

$R^{-1} \simeq \sqrt{\phi} \sin \alpha^{-1}$ and $e^{2}+\phi^{2} \simeq R K$. The $\cosh ^{2}(\sqrt{A}) \simeq \pi / e . A \simeq \sqrt{\alpha} \cosh (\pi / e)$. The $\sqrt{7 / \pi} \simeq$ $\operatorname{coth}^{2} R \simeq A R K$ [7, 32, 33]. The silver constant from the heptagon [34, 35] is approximated by $S \simeq 2 \sqrt{2} R \simeq \tan \sqrt{\phi} \simeq 3.247$. The $\sqrt{S}=2 \cos (\pi / 7) \simeq 7 \phi / 2 \pi$ and $2 \pi A \simeq S / 2 \sqrt{3} \simeq \sin 70^{\circ}$. Also relevant, $2 A \simeq S / 11 \simeq 1 / \sqrt{11}$, see the discussion of Eq. (9).

More approximations with the Great Pyramid's Golden Apex A [7, 32, 33]:

$$
A \simeq \sqrt{11} / 7 \pi \simeq \sqrt{e} / 11 \simeq \sqrt{\pi \alpha} \simeq 2 \pi \alpha S .
$$

As Jean-Paul and Robert Bauval describe in the Secret Chamber Revisited how prime numbers 7 and 11 are significant keys to the Great Pyramid, 22/7 $\simeq \pi[36]$. Also noted by David Haight, "When the Fibonacci number sequence is based on the number seven and its multiples, the Fibonacci sequence self-reflexively reappears when differences are calculated between it and this new number-seven-based Fibonacci sequence. The same thing happens with Lucas numbers." 
[10]. With the fine-structure constant, $2 \pi \alpha$ is equal to the electron Compton wavelength divided by the Bohr radius [21] and $\pi \alpha$ is the percentage of light absorbed by graphene [37]. The $\sqrt{\alpha} \simeq \sqrt{R} / 4 \pi \simeq 3 A / 2 \phi^{2}$ and $\sqrt{2 A} \simeq \sqrt{\pi} / S$. Also, $4 / \pi \simeq \sqrt{A} / 2 A \simeq \sqrt{S / 2}$ and see the Eq. (10) discussion. Finally, $\sqrt{e} / \phi \simeq 1+\alpha \phi^{2}$ [7, 32, 33].

\section{The golden ratio and fine-structure constant}

From David Haight, "prime numbers, the 'atoms' of mathematics, are necessarily related to the atoms of nature because of the well-known Rydberg rule that follows the same pattern as Euler's harmonic zeta power series (derived from the self-derived exponential growth function)." [30]. The Euler product formula for the Riemann zeta function [38, 39]:

$$
\zeta(2)=\sum_{n=1}^{\infty} \frac{1}{n^{2}}=\prod_{p \text { prime }} \frac{1}{1-p^{-2}}=\frac{\pi^{2}}{6} \simeq 1.645 .
$$

Approximations for $\zeta(2) \simeq 11 A \simeq \sqrt{7}-1$ and $\pi^{2} \simeq \sqrt{K} / 2 A \simeq 6 \sqrt{e}$. The $\ln \left(A^{-1}\right) \simeq R \zeta(2) \simeq$ $6 / \pi$, with the cube-sphere proportion. Quoting David F. Haight again, "There is geometry in the humming of the primes, there is music in the spacing of the primes." [30]. The $\sinh \sqrt{\phi} \simeq \zeta(2)$. [32, 33]. The polygon circumscribing constant $K$ is the reciprocal of the Kepler-Bouwkamp constant [40]-[42], related to "Pauli's triangle" [22], with sides approximately proportional to $1, \phi, \sqrt{\phi \sqrt{5}}$ with the golden ratio $\phi=(1+\sqrt{5}) / 2$ with $\sqrt{5}=2 \cosh (\ln \phi)$. [31]. The prime constant [32, 33], [43] is described as a binary expansion corresponding to an indicator function for the set of prime numbers. Defining the prime constant $P$ for $p(k)$ as the $k$-th prime:

$$
P=\sum_{k=1}^{\infty} 2^{-p(k)} \simeq \zeta(2) \sqrt{\alpha K} \simeq \sqrt{R A} \simeq 0.4147
$$

The prime constant, $P \simeq \sqrt{R A} \simeq \phi^{2} / 2 \pi \simeq \sqrt{3 / 2 K} \simeq \sqrt{2}-1$. Again, $K$ is the inverse Kepler-Bouwkamp constant [40]-[42]. Introduced by Arnold Sommerfeld, the fine-structure constant determines the strength of the electromagnetic interaction [44]-[49]. John S. Rigden states, "The fine-structure constant derives its name from its origin. It first appeared in Sommerfeld's work to explain the fine details of the hydrogen spectrum." [50].

Arnold Sommerfeld generalized Bohr's model to include elliptical orbits in three dimensions. He treated the problem relativistically (using Einstein's formula for the increase of mass with velocity) .... According to historian Max Jammer, this success of Sommerfeld's fine-structure formula "served also as an indirect confirmation of Einstein's relativistic formula for the velocity dependence of inertia mass. -Stephen G. Brush [51].

Charles P. Enz writes, "For Pauli the central problem of electrodynamics was the field concept and the existence of an elementary charge which is expressible by the fine-structure constant ... 1/137. This fundamental pure number had greatly fascinated Pauli .... For Pauli the explanation of the number 137 was the test of a successful field theory, a test which no theory has passed up 
to now." [52]. And again, Pauli is quoted by Varlaki, Nadai and Bokor concerning his evaluation and regard for the fine-structure constant, "The theoretical interpretation of its numerical value is one of the most important unsolved problems of atomic physics." [53].

Michael Eckert, "Even among physicists of the twenty-first century, the "Bohr-SommerfeldAtom' and the 'Sommerfeld fine-structure constant,' remain current concepts." [49]. David Haight states, "Since the ideal divergence angle in nature is the golden proportion of 137.5 degrees, could this unique proportion be the reason why 137 is the 'prime' prime number or paradigm prime in nature, beginning with hydrogen?" [10]. Harald Fritzsch recalls that "Richard Feynman, the theory wizard of Caltech in Pasadena, once suggested that every one of his theory colleagues should write on the blackboard in his office: 137 -how shamefully little we understand!" [54]. Alpha,the electromagnetic coupling constant is $\alpha=e^{2} / \hbar c$ in $\operatorname{cgs}$ units [32, 33]. Calculation of the inverse fine-structure constant as an approximate derivation from prime number theory:

$$
\alpha^{-1} \simeq 157-337 P / 7 \simeq 137.035999168 .
$$

This equation gives the same approximate value for the inverse fine-structure constant as determined in Eqs. (10-12), having three prime numbers and the prime constant [55]. Also, $\alpha^{-1} \simeq$ $\sqrt{7} \tan ^{-1}(\sqrt{\phi}) \simeq 3 / A^{2}$. The $\ln \left(A^{-1}\right) \simeq 1 / P \sqrt{\phi} \simeq \sqrt{1+\sqrt{7}} \simeq \sqrt{1+\phi^{2}}$ and $117+157 \simeq 2 \times$ 137, see discussion of Eq. (17). $337-157=180$ and the ratio 528/337 $\simeq \pi / 2$ [7], see discussion of Eq. (10). The fine-structure constant is described by David Haight as the "basis or boundary condition of the 'pre-established harmony' at the prime 'atomic' level of mathematics, physics, and cosmology, for the reason that the essences of things are numbers, especially primes." [30]. Other approximations with the Golden Apex of the Great Pyramid: $\sqrt{\alpha} \simeq A e / \phi \sqrt{K} \simeq \sqrt{R} / 4 \pi$ and $2 \pi \phi R \simeq 1 / \sqrt{\alpha}$. Also, $\ln \left(A^{-1}\right) \simeq \sqrt{K P} \approx S \sqrt{P}$ and $2 A \simeq 2 \sqrt{\pi \alpha} \simeq \phi^{2} / K \simeq \sqrt{2 / 7 \pi}$. The heptagon is a feature of the Cosmological Circle, a geometric template for the Great Pyramid and many ancient architectural designs; related to the cycloid curve and the history of the least action principle [8, 22]. From the Golden Apex $A$ and the silver constant $S, 2 A \simeq \tanh S^{-1} \simeq \tan ^{2}(1 / 2)$ , see Eq. (4).

The Wilbraham-Gibbs constant is $G_{w}$ and the sinc function, $\operatorname{sinc} x=\sin (x) / x[56]$ :

$$
G_{w}=\int_{0}^{\pi} \operatorname{sinc} x d x \simeq e \sin \alpha^{-1} \simeq K / \sqrt{7 \pi} .
$$

The Wilbraham-Gibbs constant $G_{w} \simeq \phi \ln \pi \simeq \phi^{2} / \sqrt{2} \simeq 1.852$. The Wilbraham-Gibbs constant is related to the overshoot of Fourier sums in the Gibbs phenomena [56]. Additional approximations: $G_{w} \simeq \sec (1) \simeq \exp \left(\phi^{-1}\right) \simeq \sqrt{\phi / \pi A}$, see the discussion of Eq. (18).

The inverse Kepler-Bouwkamp constant is the polygon circumscribing constant $K$ [40]-[42]:

$$
K=\frac{\pi}{2} \prod_{n=1}^{\infty} \operatorname{sinc}\left(\frac{2 \pi}{2 n+1}\right)=\prod_{n=3}^{\infty} \sec \left(\frac{\pi}{n}\right) .
$$

The polygon circumscribing constant, $K \simeq 3 / 2 R A \simeq 2 \tan (3 \pi / 7) \simeq 8.700$ and $K A \simeq \sqrt{e / \phi} \simeq$ $\sqrt{5 / 3} \simeq R+A$. Half the face apex angle of the Great Pyramid plus half the apex angle is approximately $70^{\circ}$ and $\sin \alpha^{-1} \simeq 2 \cos 70^{\circ}$ [7]. The $\csc \alpha^{-1} \simeq R \sqrt{\phi} \simeq \sqrt{7 / S} \simeq \sqrt{85} / 2 \pi$ and $528 / 504 \simeq 7 A \simeq \pi / 3$, see discussion of Eq.(10). First level sum of Teleois proportions is 85, foundational in Great Pyramid design [7] and $85 \simeq 360 / \phi^{3}$, see Eq. (11) discussion. Also, 
$85 / 11 \simeq R / A$ and $528 / 85 \simeq 2 \pi$, see Eq. (10) discussion below. The latest value for the inverse fine-structure constant by Aoyama et al, $\alpha^{-1} \simeq 137.035999157$ (41), from experimental results and quantum electrodynamics [57]. The inferred value [58] determined from quantum electrodynamic theory and experiment [59] with the least standard uncertainty in CODATA results is $\alpha^{-1} \simeq 137.035999160(33)[58]$.

$$
\sin \alpha^{-1} \simeq 504 / 85 K=7 ! /(713+137) K .
$$

Again, this equation gives $\alpha^{-1} \simeq 137.035999168$ [60]. $\sqrt{13 / 7} \simeq 2 \sin \alpha^{-1}$ and from the harmonic radii of the Cosmological Circle, $108+396=504$ [7]. 504/396 $=14 / 11 \simeq 4 / \pi$. Additionally, $\sin \alpha^{-1} \simeq \zeta(2) P \simeq 1-\pi^{-1} \simeq e / \pi \sqrt{\phi} \simeq 2 \pi / \sqrt{85} .504 / 144=7 / 2$ and $\phi \sin \alpha^{-1} \simeq$ $3 / e \simeq \sqrt{\phi} / R \simeq A e^{2}$. From Eq. (7), $\sin ^{2} \alpha^{-1} \simeq 157 / 337$. Mamombe demonstrates the link between 137 and 117 in his analysis of the Fibonacci sequence and describes "its significance to the theory of the golden section and its relationship with the fine-structure constant," [61], also see the discussion of Eq. (17) and [62]. Also, 144/85 $e / \phi \simeq 1 / 4 A$. 528/504 $\simeq 7 A$, and the Great Pyramid Key is $528 \simeq \ln (7 / A) / \alpha$ [33]. Pyramid base angle $\theta_{B} \simeq \tan ^{-1}(4 / \pi) \simeq 51.85^{\circ}$. The $\ln (4 / \pi) \simeq A \phi$ and $\pi / 4 \simeq \csc \alpha^{-1}-\sin \alpha^{-1} \simeq \sin \theta_{B} .792 / 5280=54 / 360=3 / 20 \simeq A$ and $\phi=2 \sin 54^{\circ}$ [7].

Helmut Warm found a significant ratio between the Venus/Earth synodic period and the Mars orbital period to be $17 / 20=1-3 / 20 \simeq 1 / \ln (2 \phi) \simeq \sqrt{2 \pi / K}$. This had the strongest cycle resonance found among the planets [63]. Also, 17/20=0.85, the fraction contained in the base angle of the Great Pyramid. The pyramid apex angle $\theta_{A}, \sin \alpha^{-1} \simeq \theta_{B} / \theta_{A} \simeq \sqrt{2} \tan (\pi / 7) \simeq \sqrt{S / 7} \simeq$ $2 \pi / \sqrt{85}$ [33]. Apex angle of the regular heptagon triangle is $3 \pi / 7$ and an approximation to the apex angle of the Great Pyramid. 528/396 $=4 / 3$ and 396/85 $\simeq \sqrt{S / A}$.

While twentieth-century physicists were not able to identify any convincing mathematical constants underlying the fine structure, partly because such thinking has normally not been encouraged, a revolutionary suggestion was recently made by the Czech physicist Raji Heyrovska, who deduced that the fine structure constant ... really is defined by the [golden] ratio .... - John Calleman [64].

Raji Heyrovska says, "On noticing the closeness of the fine structure constant ... to the ratio of the angles, $360^{\circ} / \phi^{2} \ldots$ the author suggested that the small difference ... could be due to the Sommerfeld's relativity correction factor." [65]. "It was also pointed out that the ratio $360^{\circ} / \phi^{2}$ ... which is a Golden section of $360^{\circ}$, differs from the inverse fine structure constant by ... $2 / \phi^{3}$ ... probably due to the difference in the g-factors for the electron and proton ... arising from the magnetic momenta of the two particles," with the result of $\alpha^{-1} \simeq\left(360 / \phi^{2}\right)-\left(2 / \phi^{3}\right)$ [66].

$$
\alpha^{-1} \simeq \frac{360}{\phi^{2}}-\frac{2}{\phi^{3}}+\frac{A^{2}}{K \phi^{4}}-\frac{A^{3}}{K^{2} \phi^{5}}+\frac{A^{4}}{K^{3} \phi^{7}} .
$$

This extension of Heyrovska's equation also gives $\alpha^{-1} \simeq 137.035999168$ [67, 68]. Raji Heyrovska found it a "surprise to find for the first time that the Bohr radius is divided into two unique sections at the point of electrical neutrality, which is the Golden point. The Golden ratio, which manifests itself in many spontaneous creations of Nature, was thus found to originate right in the core of atoms." [66]. In degrees, the modern golden angle [69], $\theta_{G}=360^{\circ} / \phi^{2}$ and related 
$\theta_{g} \simeq 26.57^{\circ} \simeq \tan ^{-1}(1 / 2)$ (twice the Cabibbo angle) is the ancient Golden Angle of Resurrection noted by Robert Temple [7, 70]. Along with the Golden Angle of Resurrection, Robert Temple states the Pythagorean Comma, $P_{c}$ was one of the greatest secrets of the ancient Egyptians [59].

Highly complex numbers like the Comma of Pythagoras, Pi and Phi (sometimes called the Golden Proportion) ... lie deep in the structure of the physical universe, and were seen by the Egyptians as the principles controlling creation, the principles by which matter is precipitated from the cosmic mind. -Jonathan Black [71].

Aubrey Meyer finds that the "Pythagorean Comma and the Fibonacci series converge on the Golden Section." [72]. For the same approximate value of $\alpha^{-1} \simeq 137.035999168$, the three terms on the right of Eq. (11) can be reduced to $\sqrt{2}(S A)^{2} / \sqrt{3} K^{2} \phi^{4} \approx 4 A^{3} / \phi^{6}$ and the last two terms can be reduced to one term with the Pythagorean Comma, $-A^{3} / \sqrt{P_{c}} K^{2} \phi^{5} . P_{c} \simeq \sqrt{2 \phi / \pi} \simeq$ $1+\sqrt{\alpha} / 2 \pi \simeq 2 \zeta(2) / S$ and $P_{c}=(3 / 2)^{12} / 2^{7} \simeq 1.014$ [7].

The fourth approximation of the inverse fine-structure constant with this particular value:

$$
\alpha^{-1} \simeq 1 / \tanh ^{-1} \tau^{-1} \simeq 137.035999168,
$$

where $\tau$ is the root of $x^{4}-137 x^{3}-6 x^{2}+98 x+7^{3}=0$, "which represents a particular quartic plane curve, different combinations of the coefficients of the general curve give rise to the lemniscate of Bernoulli." [32]. Number 7 also appears as $2 \times 7^{2}=98$ and $7^{3}=343=6+337$. The root $\tau \simeq 137.0384316101$ [73]. The other root is approximately $1.523 \simeq \pi S A \simeq \pi-\phi$.

In another representation of Eq. (12), $\operatorname{coth} \alpha \simeq \tau$. Other relations include $\tau / \cosh \phi \simeq 7 \pi / P \simeq$ $117 / \sqrt{5}$ (see the discussion of Eq. (18) below) and $\tau / \cosh ^{2} \phi \simeq 7 G_{w}+7 \simeq 7 L_{r}^{2}-7$, also from the discussion of Eq. (18). The $\cosh \phi \simeq 1 / 2 A \sqrt{\phi} \simeq P_{c} / \sqrt{A} \simeq K A\left(P_{c}+1\right) \approx \phi^{2}$. "Gauss's and Euler's study of the arc length of Bernoulli's lemniscate, a polar curve having the general form of a toric section, led to later work on elliptic functions." [32]. Also, "the lemniscate, inverse curve of the hyperbola with respect to its center, has the lemniscate constant $L$ which functions like $\pi$ does for the circle." [32, 74]. The lemniscate constant $L \simeq \cosh \phi \approx \phi^{2}$ and $\sin \alpha^{-1} \simeq$ $\sqrt{6} L / 3 \pi$, where $L / \pi$ is Gauss's constant and $\sqrt{6} / 3$ is the diameter of the inscribing sphere of the octahedron [32].

\section{Proton-electron mass ratio approximations}

With the $A$ and $K$ constants shown above, approximate values for the proton-electron mass ratio including the golden ratio and fine-structure constant:

$$
m_{p} / m_{e} \simeq A \phi^{2} / 4 \alpha^{2} \simeq 4 K / \alpha \phi^{2} .
$$

Other approximations of proton-electron mass ratio with the golden ratio and Golden Apex [7]:

$$
m_{p} / m_{e} \simeq 4(\phi+\sqrt{3}) / \alpha \simeq 2 / A \alpha .
$$

Also, $\phi \sin 70^{\circ} \simeq 2 \pi \phi A \simeq \ln \left(m_{p} / m_{e}\right) / \ln \left(\alpha^{-1}\right)$ [3, 7] and $\ln \left(\alpha m_{p} / m_{e}\right) \simeq \phi^{2} \cos A$. Malcolm H. Mac Gregor has stated, "The bridge between the electron and the other elementary particles is 
provided by the fine structure constant .... An expanded form of the constant leads to equations that define the transformation of electromagnetic energy into electron mass/energy ...." [75]. The $\exp \zeta(2) \simeq 4 \pi \cot ^{2}(1)$ and replacing $\exp \zeta(2)$ in $m_{p} / m_{e} \simeq \exp \zeta(2) / \alpha \sqrt{A}$ with $73 /(102-191 P)$ gives a more accurate value, 1836.152673485 [76]. This is approximate to the value, $m_{p} / m_{e} \simeq$ $1836.152673346(81)$, that has been derived from the most recent experimental measure of the proton mass by Heiße, F. et al. [77]. Additionally, $73+191=102+162=528 / 2=264$, related to Great Pyramid geometry in several ways [7, 33].

The half-wavelength associated with a frequency resonant to quartz crystal is 191 meters [33] and the height of the Great Pyramid without the capstone is about 137 meters: 191/137 $7 / 5 \simeq P / 2 A \simeq K / 2 \pi \simeq \sqrt{5} / \phi \simeq 528 / 382$. From the discussion of Eq. (7) $528-337=191$, $191 / 73 \simeq \phi^{2}$ and $102 / 85=6 / 5 \simeq \pi / \phi^{2}$. The length of the horizontal passage in the Great Pyramid is 102 feet [78]. $117 / 102 \simeq R$ and $117 / 73 \simeq \phi \simeq 191 / 117$. The electron/pion mass ratio, $m_{e} / m_{\pi} \simeq \alpha / 2$ and $m_{\pi} / m_{p} \simeq A$, where $m_{\pi}$ is the pion mass [32].

The omega constant [79] is defined as Lambert $W(1)$ [80]-[82], the attractive fixed point of $e^{-x} . W(1)=\Omega=\exp (-\Omega) \simeq \sinh ^{-1}(4 A) \simeq 0.5671$ and $4 A \simeq \phi / e$.

$$
\Omega=\exp (-\Omega) \simeq A \ln \left(m_{p} / m_{e}\right) / 2 .
$$

$W(x)$, the Lambert $W$-function, is an analog of the golden ratio for exponentials and is expressed as $\exp [-W(1)]=W(1)$. Also, $\Omega \simeq 2 A \ln \left(A^{-1}\right) \simeq \sqrt{\alpha} / A \simeq 1 / \sqrt{\pi}$ and $\ln \left(\alpha^{-1}\right) \simeq K \Omega$. From the zeta function, $\zeta(2) / \Omega \simeq 1+\ln \left(A^{-1}\right)$.

In the Foundation Stone of classical harmonics the alpha harmonic is equal to the sum of the golden ratio harmonic and the omega constant harmonic, relating the ancient Greek Pythagorean form of metaphor for the fine-structure constant to golden ratio geometry and architecture [32].

\section{Lucas numbers and golden ratio geometry}

The reciprocal Lucas constant is $L_{r} \simeq 1.9628$ [81, 82] with a derivation from the golden ratio:

$$
L_{r}=\sum_{n=1}^{\infty} \frac{1}{L_{n}}=\sum_{n=1}^{\infty} \frac{1}{(-\phi)^{-n}+\phi^{n}} .
$$

Approximately, $L_{r} \simeq 1 / \sqrt{A \sqrt{3}} \simeq 2 \sqrt{K} / 3 \simeq \sqrt{\phi} \cosh (1) \simeq \sqrt{e / \phi} / \lambda$, where $\lambda$ is the Laplace limit [85] of Kepler's equation described previously with the fine-structure constant [32]. Also, $\operatorname{coth} \lambda^{-1} \simeq \phi \sin \alpha^{-1}$, see the discussion of Eq. (10) and Eq. (18).

$$
L_{r} \simeq P / \sqrt{2} A \simeq S / 4 P \simeq \sqrt{2 / \phi} / \Omega .
$$

The $\cosh ^{-1} L_{r} \simeq \sqrt{e / \phi} \simeq K A$ and $\ln \left(m_{p} / m_{e}\right) \simeq \Omega L_{r} / A$. The reciprocal Lucas constant $L_{r} \simeq$ $-\tan 117^{\circ} \simeq 3 \phi^{2} / 4$, the square of the radius of the circumscribing sphere of the dodecahedron. The golden ratio $\phi \simeq A \sqrt{117}$ and $\sinh ^{-1}(117) \simeq \phi / 2 A$. Also, $137 \simeq 117 \sinh (1)$ and $117 \simeq 2 K / A \simeq 10 / \sqrt{\alpha}$. The approximate dihedral angle of the dodecahedron $117^{\circ} \simeq 180^{\circ}-$ $\tan ^{-1}(2) \simeq L_{r} \tan ^{-1}\left(\phi^{-1}\right) / T$, see Eq. (18) for Tau $T$. Also, the Pythagorean relation $117^{2}+$ $137^{2} \simeq 108^{2}+144^{2}=180^{2}$ and $144 / 117 \simeq 2 / \phi$ [22]. With the Great Pyramid Key 528 again, $528 / 137 \simeq L_{r}^{2}$ and $137 / 21 \simeq L_{r}^{2} / 4 A$; related to the hydrogen emission resonance of the King's Chamber [1]. 
Regarding the golden ratio again, Boeyens and Thackeray [86] are quoted by Mamombe [61], "We suggest that there is a strong case that the so-called, 'Golden Ratio' (1.61803 ...) can be related not only to aspects of mathematics but also to physics, chemistry, biology and the topology of space-time." [86]. Xu and Zhong state, “... we would like to draw attention to a general theory dealing with the noncommutativity and the fine structure of spacetime which comes to similar conclusions and sweeping generalizations about the important role which the golden mean must play in quantum and high energy physics." [87]. In the Fibonacci inspired sequences studied by Mamombe the number 117 marks a transition point, which is also a harmonic of the square root of the inverse fine-structure constant [48] and he describes a "geometrical basis for the fine-structure constant in the golden section." [61].

The Egyptian Royal Cubit is a traditionally known measure basis for the Royal Cubit and the numerical harmonic of the meter is also found in the Great Pyramid geometry, $\pi / 6 \simeq \phi^{2} / 5 \simeq \pi-$ $\phi^{2} \simeq 0.5236$ meters, which is approximately equal to a Royal Cubit [33]. $L_{r} \simeq(T+\sqrt{7}) / \phi \simeq$ $2 T \phi \ln \pi, \cosh T \simeq \ln \pi$ and Tau $T$ is described as:

$$
T=\alpha+\pi / 6 \simeq 2 A / \Omega \simeq \ln \left(L_{r}\right) / \sqrt{\phi} .
$$

$T \simeq \sqrt{7} / 5 \simeq 2 \pi \sqrt{\alpha} \simeq 0.5309$. From the Great Pyramid, with a height of 280 Royal Cubits, $280 / 117 \simeq \sqrt{7 \pi} / L_{r} \simeq 2.4$ is the golden angle in radians or $360^{\circ} / \phi^{2} \simeq 137.5^{\circ} .117^{\circ} \simeq \theta_{G}(1-A)$ with $\theta_{G}$ in degrees. The modern golden angle, $\theta_{G} \simeq \sqrt{\phi} / T \simeq \ln \left(A^{-1}\right)+1 / 2 \simeq 2.4$ radians, see the discussion of Eq. (11). Also, $T \simeq \sqrt[3]{A} \approx 2 A \sqrt{\pi} \simeq L_{r} / 2 G_{w} \simeq L_{r}(1-A) / \pi \simeq \phi^{2} / \ln \left(\alpha^{-1}\right) \simeq$ $L_{r} \sqrt{\phi / 7 \pi} \simeq \cos 58^{\circ}$. The base of the face angle on the Great Pyramid is approximately $58^{\circ}$. Manu Seyfzadeh explains that "The pyramid's core design is based on a Kepler Triangle whose hypotenuse (356 cubits) is in the golden ratio $(1.618 \ldots)$ to its short leg (220 cubits) incidentally producing a side-slope angle of $51^{\circ} 50^{\prime} . "$ [88]. $T / \phi \simeq 117 / 356$.

The ratio of angles, $117^{\circ} / 51.85^{\circ} \simeq \sqrt{\phi \pi} ; \phi \pi$ is another frequently encountered measure in the Great Pyramid. 356/70 $\varnothing \pi$, from another Kepler Triangle. Seyfzadeh says, "Two classic interrelated geometric relationships can be approximated from an observable measure of Earth's curvature and a sidereal lunar year." [88]. With another significant number from the Great Pyramid measures $432,432 / 117 \simeq 6 / \phi \simeq L_{r} / T$. Also, $R / T \simeq 2 S / 3 \simeq 2 \zeta(4)$ and $R T \simeq 1 / \zeta(2)$. $70 / 117 \simeq 4 A$ and $K A \simeq T \sqrt{6}$.

The Royal Cubit again, $\pi / 6=\zeta(2) / \pi \simeq T / P_{c}$, where $P_{c}$ is the Pythagorean Comma from the discussion of Eq. (11). Also, $6 / \pi \simeq P K T \simeq \sqrt{1+\sqrt{7}}$. From tetrahedral geomery, $\cos 19.5^{\circ} \simeq$ $\sin (6 / \pi)$, in radians. From Great Pyramid geometry, $\sin ^{-1} \phi^{-2} \simeq 69.4^{\circ}+90^{\circ}-137^{\circ}$ and the $\tan 69.4^{\circ} \simeq \phi \zeta(2) \simeq \sqrt{2} / T \simeq \tan \left(\phi^{2} \theta_{g}\right)$, see the discussion of Eq. (11). The $\ln \left(L_{r} / T\right) \simeq \phi^{2} / 2$. $T^{-1} \simeq 4 \pi A \simeq \zeta(2) \ln \pi \simeq \sqrt{\phi}+\phi^{-1} \simeq L_{r}^{2}-L_{r}, \sqrt{T} \simeq 1 / L_{r}(1-2 A) \simeq 2 \pi / K$ and $\sqrt[3]{T} \simeq \phi / 2$. $T / \Omega \simeq 2 \pi A$ and $\Omega T \simeq 2 A \simeq K A-1$.

The Royal Cubit in inches, $144 / 7 \simeq R_{c}$ [89]. Alpha, $\alpha \simeq A / R_{c}$, related to the symbolism in historical references concerning the fine-structure constant [90]. Multiplying, $\alpha \times \Omega \times T \simeq$ $1 / 7 \pi R_{c}$. Adding, $\alpha+\Omega+T \simeq \sqrt{\phi} / R$, see the discussion of Eq. (10). Also, $A+\Omega+T \simeq$ $2 \sqrt{140 / 360}=\sqrt{14} / 3 \simeq \phi \tan (\pi-\phi) \simeq \phi \sqrt{\phi / e} \simeq \sqrt{2} / 2 \Omega \simeq R_{c} / 2 \lambda_{H}$, with $R_{c}$ and $\lambda_{H}(21 \mathrm{~cm}$ hydrogen emission wavelength) in meters and $T \simeq P_{c} R_{c}$. From the hieroglyph for gold, 14/9 $\simeq$ $\tan (1)$ and $A+\Omega \simeq 9 / 4 \pi \simeq \sqrt{\phi / \pi}$ [7, 22]: "The vertex angle of the nonagon, $140^{\circ}$, when considered as the central angle of a triangle in a circle forms the Egyptian hieroglyph for neb or 
gold related to quintessence, or the 'unified field' of physics." [22].

These suggestive references to the quintessential aether are interpretations of Herschel's AlphaOmega-Taurus Star Gate [91], the Ark of Osiris from Coppens [92] and Hardy's Pyramid Energy [93]. Inverse Golden Apex, $A^{-1}$ as a harmonic of the Earth frequency utilized by Tesla of 6.67 $\mathrm{Hz}$ [94], divided by Tesla's magnifying transmitter frequency of $11.78 \mathrm{~Hz}: 6.67 / 11.78 \simeq \Omega$. With Parr's 51.5 Hz pyramid orb frequency, the ratio is $51.5 / 11.78 \simeq 2 \pi-(6 / \pi) \simeq 6 \sqrt{T} \simeq K / 2$ [95, 96].

\section{Conclusion}

Euler's equation and the exponential function applied to the geometry of the Golden Apex of the Great Pyramid were part of four different calculations of the inverse fine-structure constant with the same approximate value. The fine-structure constant and the Golden Apex were related to the proton-electron mass ratio, golden ratio and other fundamental constants; finally speculating briefly on the metaphorical physics and mathematical metaphysics of the Great Pyramid [97, 98].

These results illustrate clearly the highly interrelated nature of the fundamental constants. Dependent as our knowledge of them is upon many different fields of physics, we have here a good example of the importance of making occasional analyses of the consistency situations of sufficiently inclusive scope to serve as valuable guides to further research. The present example emphasizes especially that a better knowledge of the Sommerfeld constant, $\alpha$, would be of great value to physics at the present time. -Jesse DuMond [99].

David Haight quotes "Physicist John Wheeler, who coined the term 'geometrodynamics' put it this way, 'Physics is really geometry .... Some profound connection exists between the fundamental constants of microphysics and the geometry of the cosmos." [10].

This unification of mathematics through Phi should not come as a complete surprise to us since Phi is related to all three means that are essential to mathematics-the arithmetic, the geometric and the harmonic. (These three means are the result of the calculus of differences, just as the harmonic intervals in music are the result of the calculus of variations.) -David Haight [10].

Eckhart Schmitz emphasizes, "The Great Pyramid of Giza may clearly be regarded as a repository of ancient knowledge." [12]. In conclusion, Witteveen is quoted again on Pythagoras, "All is number, everything is movement, everything is music in the harmony of the spheres." [1]. This 'harmony of the spheres' is exemplified by the Golden Apex [7] in a relation which also 'squares the circle' [1], $\sqrt{A / 2} \simeq 3 / 11$. As Robin Heath explains, "This ratio of 3:11 is exactly the ratio between the Moon's diameter ... and the diameter of the mean Earth (the first major treatment of this was in City of Revelation by John Michell [100], who was responsible for its rediscovery)." [3]. With reference to the revelation of this harmony Mary Anne Atwood states, "And here the external and internal worlds were seen to blend together in confluent harmony, proving and establishing each other, and leaving reason nothing more to doubt of, or the senses to desire, but a fulfillment under the universal law. [101]. 


\section{Acknowledgments}

Special thanks to Case Western Reserve University, PhilPapers, MathWorld and WolframAlpha.

\section{References}

[1] Witteveen, W. The Great Pyramid of Giza: A Modern View on Ancient Knowledge, Kempton, IL: Adventures Unlimited Press, 2016, 247, 96, 239.

[2] Vries, M. The Whole Elephant Revealed: Insights into the Existence and Operation of Universal Laws and the Golden Ratio, Hampshire, UK: Axis Mundi Books, 2012, 370.

[3] Heath, R. Sacred Number and the Origins of Civilization: The Unfolding of History through the Mystery of Number, New York, NY: Simon and Schuster, 2006, 132.

[4] Livio, M. The Golden Ratio: The Story of Phi, the World's Most Astonishing Number, New York, NY: Crown Archetype, 2008, 6.

[5] Stakhov, A. "A History, the Main Mathematical Results and Applications for the Mathematics of Harmony," Applied Mathematics, 5, 3, 363-386 (2014). am.2014.53039.

[6] Stakhov A. "Dirac's Principle of Mathematical Beauty, Mathematics of Harmony and "Golden" Scientific Revolution," Visual Mathematics, 11, 1 (2009). vismath/stakhov

[7] Sherbon, M.A. "Quintessential Nature of the Fine-Structure Constant," Global Journal of Science Frontier Research A, 15, 4, 23-26 (2015). hal-01174786v1.

[8] Sherbon, M.A. "Nature's Information and Harmonic Proportion," SSRN Philosophy of Science eJournal, 5, 3 (2011). doi/10.2139/ssrn.1766049.

[9] Olsen, S. The Golden Section: Nature's Greatest Secret, New York, NY: Walker Publishing Company, 2006.

[10] Haight, D.F "A Novel Way to Construct the Fibonacci Sequence and the Uni-Phi-cation of Mathematics and Physics," Pure and Applied Mathematics Journal, 4, 4, 139-146 (2015). doi/10.11648/j.pamj.20150404.11

[11] Gazalé, M.J. Gnomon: From Pharaohs to Fractals, Princeton, NJ: Princeton University Press, 1999.

[12] Schmitz, E.R. The Great Pyramid of Giza: Decoding the Measure of a Monument, Ontario, CA: Roland Publishing, 2012, 3, 89.

[13] Primrose, A.C. The Lamb Slain with a Golden Cut: Spiritual Enlightenment and the Golden Mean Revelation, Bloomington, IN: Balboa Press, 2016, 99.

[14] Eisen, W. "The Great Pyramid of the Emperor," in The Essence of the Cabalah, Marina Del Rey, CA: DeVorss, 1984, 474-479. 
[15] Eisen, W. "The Mysteries of the Constants (e) and (i)," in The Universal Language of Cabalah, Marina Del Rey, CA: DeVorss, 1989, 160-178.

[16] Eisen, W. The English Cabalah Volume I: The Mysteries of Pi, Marina Del Rey, CA: DeVorss, 1980.

[17] Eisen, W. The English Cabalah Volume II: The Mysteries of Phi, Marina Del Rey, CA: DeVorss, 1982.

[18] Richard Feynman, QED: The Strange Theory of Light and Matter, Princeton, NJ: Princeton University Press, 1985.

[19] Weisstein, E.W. "Exponential Function," MathWorld-A Wolfram Web Resource. mathworld.wolfram.com/ExponentialFunction

[20] Weisstein, E.W. "e Approximations." MathWorld-A Wolfram Web Resource. mathworld.wolfram.com/eApproximations

[21] Rosen, S.M. "Pauli's Dream: Jung, Modern Physics and Alchemy," Quadrant, 44, 2, 49-71 (2014). $\mathrm{rg}-22770700$.

[22] Sherbon, M.A. "Wolfgang Pauli and the Fine-Structure Constant," Journal of Science, 2, 3, 148-154 (2012). hal-01304518v1.

[23] Rosen, S.M. "Quantum Gravity and Phenomenological Philosophy," Foundations of Physics, 38, 556-582, (2008). s10701-008-9221-5.

[24] Hu, H. \& Wu, M. "Spin as Primordial Self-Referential Process Driving Quantum Mechanics, Spacetime Dynamics and Consciousness," NeuroQuantology, 2, 1 (2007). nq.2004.2.1.35.

[25] Rapoport, D.L. "Surmounting the Cartesian Cut through Philosophy, Physics, Logic, Cybernetics, and Geometry: Self-Reference, Torsion, the Klein Bottle, the Time Operator, Multivalued Logics and Quantum Mechanics," Foundations of Physics, 41, 1, 33-76 (2011). s10701-009-9334-5.

[26] Irwin, K. et al, "Quantum Walk on Spin Network and the Golden Ratio as the Fundamental Constant of Nature," Proceedings of the Fourth International Conference on the Nature and Ontology of Spacetime, C16-05-30.9, 117-160 (2017). arXiv:1602.07653v3 [hep-th].

[27] Pauli, W. "Modern Examples of Background Physics," in Meier, C.A. ed. Atom and Archetype, Princeton, NJ: Princeton University Press, 2001.

[28] Pauli, W. "The Influence of Archetypal Ideas on the Scientific Theories of Kepler," in Enz, C.P. \& Meyenn, K. eds. Wolfgang Pauli: Writings on Physics and Philosophy, New York: Springer, 1994, pp.219-279. 
[29] Gieser, S. The Innermost Kernel: Depth Psychology and Quantum Physics: Wolfgang Pauli's Dialogue with C.G. Jung, Berlin: Springer, 2005.

[30] Haight, D.F. "Why the Glove of Mathematics Fits the Hand of the Natural Sciences So Well: How Far Down the (Fibonacci) Rabbit Hole Goes," European Scientific Journal, 12, 15 (2016). eujournal.org/index.php/esj/article/view/7488.

[31] Weisstein, E.W. "Golden Ratio," MathWorld-A Wolfram Web Resource. mathworld.wolfram.GoldenRatio.

[32] Sherbon, M.A. "Fundamental Nature of the Fine-Structure Constant," International Journal of Physical Research, 2, 1, 1-9 (2014). hal-01304522v1.

[33] Sherbon, M.A. "Fundamental Physics and the Fine-Structure Constant," International Journal of Physical Research, 5, 2, 46-48 (2017). hal-01312695v1.

[34] Weisstein, E.W. "Silver Constant," MathWorld-A Wolfram Web Resource. mathworld.wolfram.com/SilverConstant.

[35] Weisstein, E.W. "Heptagon," MathWorld-A Wolfram Web Resource. mathworld.wolfram.com/Heptagon.

[36] Bauval, R. Secret Chamber Revisited: The Quest for the Lost Knowledge of Ancient Egypt, Rochester, VT: Bear \& Company, 2014.

[37] Nair, R.R. et al. "Fine Structure Constant Defines Visual Transparency of Graphene," Science, 320, 5881, 1308-1308 (2008). arXiv:0803.3718v1 [cond-mat.].

[38] Sondow, J. \& Weisstein, E.W. "Euler Product," MathWorld-A Wolfram Web Resource. mathworld.wolfram.com/EulerProduct.

[39] Sondow, J. \& Weisstein, E.W. "Riemann Zeta Function," MathWorld-A Wolfram Web Resource. mathworld.wolfram.com/RiemannZetaFunction,

[40] Finch, S.R. "Kepler-Bouwkamp Constant," Mathematical Constants, Cambridge, UK: Cambridge University Press, 2003, 428-429.

[41] Sloane, N. J. A. Sequence A051762 "Polygon Circumscribing Constant," in The On-Line Encyclopedia of Integer Sequences. oeis.org/A051762.

[42] Weisstein, E.W. "Polygon Circumscribing," MathWorld-A Wolfram Web Resource. mathworld.wolfram.com/PolygonCircumscribing.

[43] Weisstein, E.W. "Prime Constant," MathWorld-A Wolfram Web Resource. mathworld.wolfram.com/PrimeConstant.

[44] Mac Gregor, M.H. The Power of Alpha, Hackensack, NJ: World Scientific, 2007.

[45] Barrow, J.D. The Constants of Nature: From Alpha to Omega-the Numbers That Encode the Deepest Secrets of the Universe, New York, NY: Pantheon Books, 2002. 
[46] Miller, A.I. Deciphering the Cosmic Number, New York, NY: W.W. Norton, 2009.

[47] Kragh, H. "Magic Number: A Partial History of the Fine-Structure Constant," Archive for History of Exact Sciences, 57, 5, 395-431 (2003). s00407-002-0065-7

[48] Kragh, H. Niels Bohr and the Quantum Atom: The Bohr Model of Atomic Structure 19131925, Oxford, UK: Oxford University Press, 2012.

[49] Eckert, M. Arnold Sommerfeld: Science, Life and Turbulent Times 1868-1951, New York, NY: Springer, 2013, xi.

[50] Rigden, J.S. Hydrogen: The Essential Element, Cambridge, MA: Harvard University Press, 2003, 55.

[51] Brush, S.G. Making 20th Century Science: How Theories Became Knowledge, Oxford, UK: Oxford University Press, 2015, 220.

[52] Pauli, W. \& Enz, C.P. Pauli Lectures on Physics: Volume 1, Electrodynamics, North Chelmsford, MA: Courier Corporation, 2000, x.

[53] Varlaki, P., Nadai, L. \& Bokor, J. "Number Archetypes and 'Background' Control Theory Concerning the Fine-Structure Constant," Acta Polytechnica Hungarica, 5, 2, 71-104 (2008).Varlaki_Nadai_Bokor_14.pdf.

[54] Fritzsch, H. Elementary Particles: Building Blocks of Matter, Hackensack, NJ: World Scientific, 2005, 58.

[55] Sherbon, M.A. Fine-Structure Constant Calculation of Eq.(7) from WolframAlpha. WolframAlpha/Fine-StructureConstant

[56] Zi-Xiang, Z. "An Observation of Relationship Between the Fine Structure Constant and the Gibbs Phenomenon in Fourier Analysis," Chinese Physics Letters, 21.2, 237-238 (2004). arXiv:0212026v1 [physics.gen-ph].

[57] Aoyama, T., Hayakawa, M., Kinoshita, T. \& Nio, M. "Tenth-Order Electron Anomalous Magnetic Moment: Contribution of Diagrams without Closed Lepton Loops," Physical Review D, 91, 3, 033006 (2015). arXiv:1412.8284v3 [hep-ph].

[58] Mohr, P.J., Newell, D.B. \& Taylor, B.N. "CODATA Recommended Values of the Fundamental Physical Constants: 2014," Reviews of Modern Physics, 88, 035009 (2016). doi/10.1103/RevModPhys.88.035009

[59] Hanneke, D., Fogwell, S. \& Gabrielse, G. "New Measurement of the Electron Magnetic Moment and the Fine Structure Constant," Physical Review Letters, 100, 120801 (2008) arXiv:0801.1134v2 [physics.atom-ph].

[60] Sherbon, M.A. Fine-Structure Constant Calculation of Eq.(10) from WolframAlpha. WolframAlpha/Fine-StructureConstant 
[61] Mamombe, L. "Proportiones Perfectus Law and the Physics of the Golden Section," Asian Research Journal of Mathematics, 7, 1, 1-41 (2017). doi/10.9734/arjom2017/36860.

[62] Mamombe, L. "From Pascal Triangle to Golden Pyramid," Asian Research Journal of Mathematics, 6, 2, 1-9 (2017). doi/10.9734/arjom/2017/29964.

[63] Warm, H. Signature of the Celestial Spheres: Discovering Order in the Solar System, Forest Row, UK: Rudolf Steiner Press, 2010, 130.

[64] Calleman, C.J. The Purposeful Universe: How Quantum Theory and Mayan Cosmology Explain the Origin and Evolution of Life, New York, NY: Simon and Schuster, 2009.

[65] Heyrovska, R. "The Golden Ratio, Ionic and Atomic Radii and Bond Lengths," Molecular Physics, 103, 877 - 882 (2005). doi/10.1080/00268970412331333591.

[66] Heyrovska, R. "Golden Ratio Based Fine Structure Constant and Rydberg Constant for Hydrogen Spectra," International Journal of Sciences, 2, 5, 28-31 (2013). ijsciences.com/pub/article/185.

[67] Sherbon, M.A. Fine-Structure Constant Calculation of Eq.(11) Part 1 from WolframAlpha. WolframAlpha/input1.

[68] Sherbon, M.A. Fine-Structure Constant Calculation of Eq. (11) Part 2 from WolframAlpha. WolframAlpha/input2.

[69] Weisstein, E.W. "Golden Angle," MathWorld-A Wolfram Web Resource. mathworld.wolfram.com/GoldenAngle.

[70] Temple, R.G. Egyptian Dawn: Exposing the Real Truth Behind Ancient Egypt, London, UK: Century, 2010.

[71] Black, J. The Secret History of the World, London, UK: Quercus Publishing, 2013.

[72] Meyer, A. "Math, Music \& Nature's Derivation of Phi," Portmeirion Festival Presentation (03-09-2016). gci.org.uk/PEB.html.

[73] Sherbon, M.A. Fine-Structure Constant Calculation of Eq. (12) from WolframAlpha. wolframalpha.com/input.

[74] Weisstein, E.W. "Lemniscate Constant," MathWorld-A Wolfram Web Resource. mathworld.wolfram.com/Lemniscate.html.

[75] Mac Gregor, M.H. The Enigmatic Electron: A Doorway to Particle Masses, Santa Cruz, CA: El Mac Books, 2013.

[76] Sherbon, M.A. Proton-Electron Mass Ratio Calculation from WolframAlpha. WolframAlpha/massratio.

[77] Heiße, F. et al. "High-Precision Measurement of the Proton's Atomic Mass," Physical Review Letters, 119, 3, 033001 (2017). arXiv:1706.06780v1 [physics.atom-ph]. 
[78] Smith, W. Miracle of the Age: The Great Pyramid of Gizeh, Pomeroy, WA: Health Research Books, 1996, 72.

[79] Weisstein, E.W. "Omega Constant," MathWorld-A Wolfram Web Resource. mathworld.wolfram.com/OmegaConstant.

[80] Weisstein, E.W. "Lambert W-Function," MathWorld-A Wolfram Web Resource. mathworld.wolfram.com/LambertW-Function.

[81] Valluri, S.R, Jeffrey, D.J. \& Corless, R.M. "Some Applications of the Lambert W Function to Physics," Canadian Journal of Physics, 78, 9, 823-831 (2000) doi/10.1139/p00065 .

[82] Sloane, N. J. A. Sequence A030178: "Decimal expansion of Lambert W(1)," in The OnLine Encyclopedia of Integer Sequences. oeis.org/A030178.

[83] Weisstein, E.W. "Reciprocal Lucas Constant," MathWorld-A Wolfram Web Resource. mathworld.wolfram.com/ReciprocalLucasConstant.

[84] Brousseau, A. Fibonacci and Related Number Theoretic Tables, Santa Clara, CA: The Fibonacci Association, 1972, 45.

[85] Weisstein, E.W. "Laplace Limit," MathWorld-A Wolfram Web Resource. mathworld.wolfram.com/LaplaceLimit.

[86] Boeyens, J.C. \& Thackeray, J.F. "Number Theory and the Unity of Science," South African Journal of Science, 110, 11-12 (2014). sajs.2014/a0084.

[87] Xu, L. \& Zhong, T. "Golden Ratio in Quantum Mechanics," Nonlinear Science Letters B: Chaos, Fractal and Synchronization, 1, 10-11 (2011). xu-zhong.pdf.

[88] Seyfzadeh, M. "The Mysterious Pyramid on Elephantine Island: Possible Origin of the Pyramid Code," Archaeological Discovery, 5, 187-223 (2017). doi/10.4236/ad.2017.54012,

[89] Nightingale, E.G. \& Scranton, L. (Afterword) The Giza Template: Temple Graal Earth Measure, Bangor, PA: Nightingale, 2014.

[90] Varlaki, P. et al, "Historical Origin of the Fine Structure Constant, Parts I-III," Acta Polytechnica Hungarica, 7, 1, 119-157 (2010) 8, 2, 161-196; 8, 6, 43-78 (2011). rgvarlaki_rudas_koczy.

[91] Herschel, W. The Alpha-Omega-Taurus Star Gate: The Hidden Records Chronicles, Melkbosstrand Cape, ZA: THRBooks, 2017.

[92] Coppens, P. The Canopus Revelation: The Stargate of the Gods and the Ark of Osiris, Kempton, IL: Adventures Unlimited Press, 2004.

[93] Hardy, D. et al, Pyramid Energy: The Philosophy of God, the Science of Man, Clayton, GA: Cadake Industries, 1987. 
[94] Puharich, A. "Effects of Tesla's Life \& Inventions," in Valone, T. Harnessing the Wheelwork of Nature: Tesla's Science of Energy, Kempton, IL: Adventures Unlimited Press, 2002, 122.

[95] Parr, J. "The Mystery and Secret of the Great Pyramid," Great Pyramid of Giza Research Association (2009). gizapyramid.com/Parr/Index2.html.

[96] Davidson, D. "Experimental Research on Shape Power Energies," in DeSalvo, J. The Complete Pyramid Sourcebook, Bloomington, IN: 1stBooks, 2003, 289-304.

[97] Lubicz, R.A.S. Symbol and the Symbolic: Ancient Egypt, Science and the Evolution of Consciousness, New York, NY: Inner Traditions International, 1982.

[98] Lubicz, R.A.S. Sacred Science: The King of Pharaonic Theocracy, New York, NY: Inner Traditions International, 1988.

[99] DuMond, J.W.M. "The Present Key Importance of the Fine Structure Constant, $\alpha$, to a Better Knowledge of All the Fundamental Physical Constants," Journal for Nature Research, A 21, 1-2, 70-79 (1966). Online (2014). doi/10.1515/zna-1966-1-211.

[100] Michell, J. City of Revelation: On the Proportion and Symbolic Numbers of the Cosmic Temple, London, UK: Garnstone Press, 1972.

[101] Atwood, M.A. A Suggestive Inquiry into the Hermetic Mystery, London, UK: J.M. Watkins, 1918, 416. 